\title{
Safety and efficacy of a $100 \%$ dimethicone pediculocide in school-age children
}

\author{
Erin Speiser Ihde ${ }^{1 *}$, Jeffrey R. Boscamp ${ }^{2}$, Ji Meng Loh ${ }^{3}$ and Lawrence Rosen
}

\begin{abstract}
Background: Head lice most commonly affect children, ages 3 to 11. Concerns exist about the safety and efficacy of pesticide-based treatments. Published studies suggest dimethicone is a potentially safe and effective non-toxic treatment, but have not evaluated $100 \%$ dimethicone in a pediatric population. The objectives were to evaluate the efficacy and safety of $100 \%$ dimethicone for the treatment of head lice in children, monitored by school nurses.

Methods: This was a multi-site, open-label study of a $100 \%$ dimethicone gel for the treatment of head lice in a pediatric population. Children (ages 3-12) suspected of infestation with head lice were evaluated by school nurses at six schools and daycare programs in New York and New Jersey. Inclusion criteria were presence of at least three live lice, or one live louse and 10 viable eggs (eggs found within $1.27 \mathrm{~cm}$ of the scalp) and no use of any head lice treatment within four weeks of enrollment. Counts of live lice and viable eggs found in 58 subjects were tracked at baseline (Day 0) and on Day 1, Day 7, and Day 14 after treatment.
\end{abstract}

Results: After 1 day of treatment with $100 \%$ dimethicone, $98.30 \%$ of subjects were free of live lice and $55.20 \%$ were free of viable eggs. On day $14,96.50 \%$ were still free of live lice, and $80.70 \%$ were free of viable eggs. All subjects were monitored by the school nurse at baseline and throughout the study period for adverse effects, including scalp erythema, excoriation, flaking and edema. There was one adverse event of skin irritation lasting $10 \mathrm{~min}$, and no serious adverse events reported. Overall, scalp conditions improved from the baseline: 10 subjects $(17.5 \%)$ reported mild to moderate scalp erythema on day 1 , compared with only one subject (1.7 \%) on day 14 ; 8 subjects (14.3\%) reported mild scalp excoriation on day 1, with none reporting on day 14 .

Conclusions: $100 \%$ dimethicone was found to be a safe and highly effective treatment for pediatric head lice. Because dimethicone avoids pesticide exposure and resistance issues, dimethicone should be considered as a first-line treatment for head lice.

Trial Registration: NCT02213055 Date of registration: August 8, 2014

Standards of reporting: The CONSORT 2010 Checklist was consulted during the review of this manuscript. Please note that sections pertaining specifically to randomized controlled trials (RCT's) were not applicable.

Keywords: Pediculosis, Pediculus humanus capitis, Pediatrics, Head lice, Dimethicone, Infestation, School, Children

\footnotetext{
* Correspondence: eihde@HackensackUMC.org

${ }^{1}$ The Deirdre Imus Environmental Health Center ${ }^{\circledast}$, Hackensack University

Medical Center, 30 Prospect Ave, Hackensack, NJ 07601, USA

Full list of author information is available at the end of the article
}

\section{Ciomed Central}

(c) 2015 Ihde et al. This is an Open Access article distributed under the terms of the Creative Commons Attribution License (http://creativecommons.org/licenses/by/4.0), which permits unrestricted use, distribution, and reproduction in any medium, provided the original work is properly credited. The Creative Commons Public Domain Dedication waiver (http:// creativecommons.org/publicdomain/zero/1.0/) applies to the data made available in this article, unless otherwise stated. 


\section{Background}

Head lice, or Pediculus humanus capitis, cause an estimated 6 to 12 million infestations per year in the U.S., most commonly affecting children 3 to 11 years of age [1]. Head lice affect all socio-economic groups. Infestations spread regardless of the cleanliness of a home or school environment, or of personal hygiene. It is a significant public health issue due to the high anxiety for children and parents associated with infestation, as well as missed school days. Despite the American Academy of Pediatrics' recommendation not to restrict infested children from school, there are districts that continue to have a no-nit policy and guardians who keep children out of school [2].

The most common treatments are pesticide-based, over-the-counter remedies containing permethrin (1\%) or other pyrethroid insecticides. Prescription-only options include high-dose permethrin (5\%), malathion (0.5\%), benzyl alcohol lotion (5\%), ivermectin lotion $(0.5 \%)$ and spinosad suspension $(0.9 \%)$ [3].

Pesticide-based treatments are coming under increasing scrutiny regarding safety and efficacy. Specifically, exposures to neurotoxic pesticides have been linked to lowered IQ, diminished attention span, other neurodevelopmental issues and childhood cancers [4-6]. Additionally, studies have found insecticide resistance in head lice, [7-12] particularly to permethrin and malathion. Given these concerns, an effective and safe alternative pesticide-free treatment is desirable.

Dimethicone (also spelled dimeticone) is a siliconebased polymer that works mechanically to lubricate hair to aid the removal of nits and lice, while physically occluding the respiratory system of the louse. Burgess found that dimethicone potentially eliminates the pesticide-resistance issue as "the blockage [of dimethicone coating the louse] inhibits water excretion, which causes physiological stress that leads to death either through prolonged immobilisation or, in some cases, disruption of internal organs such as the gut" [13].

Several published trials have established plausibility for the use of dimethicone in pediatric lice treatment, demonstrating it may be more effective than conventionallyrecommended pesticide-based pediculocides [13-26]. These studies include concentrations of 4-96 \% dimethicone in vivo. Only one published trial evaluated the in vitro ovicidal efficacy of $100 \%$ dimethicone. No previously published study has examined the in vivo safety and efficacy of a $100 \%$ dimethicone-based treatment for head lice. Dimethicone-based treatments are commonly used in the European Union and even recommended as a first-line treatment in some Australian jurisdictions [27]. However, they are not a first line treatment in the U.S. and are not listed as one of the recommended treatments in the American Academy of Pediatrics' (AAP) 2012 Red Book $^{\ominus}$ [28]. Dimethicone is listed in the AAP's 2015 Clinical Report as an alternative treatment [29].
The objectives of this study were to evaluate the efficacy and safety of $100 \%$ dimethicone gel for the topical treatment of head lice in a pediatric, school-centered population.

\section{Methods}

This was a multi-site, open-label study of a $100 \%$ dimethicone gel [LiceMD, manufactured by Combe, Inc, subsequently renamed as LiceMD Pesticide Free] for the treatment of head lice in children. The $100 \%$ dimethicone liquid has a viscosity of 350 centistokes and is composed of a single viscosity polymer.

Children (ages 3-12) suspected of infestation with head lice were evaluated from May 2009 to June 2013 by school nurses at six schools and daycare programs in New York and New Jersey who were trained in all study procedures. Subjects were referred to the school nurse for head lice evaluation by school staff members, parents/ guardians, or were diagnosed as part of routine school head checks. Written informed consent for participation in the study was obtained from the subject's parent or guardian. Additionally, each child nine years of age or older was asked to read and sign an assent form if he or she agreed to participate in the study.

Children could be included as subjects if they met the following criteria as determined by the school nurses, who were trained in how to detect lice using a standard visual census technique: presence of at least three live lice or one live louse and 10 viable eggs (eggs found within $1.27 \mathrm{~cm}$ of the scalp). The subject could not have used any head lice treatment within four weeks of enrollment, as reported to the nurse by the parent/guardian. A child could be enrolled more than once in the study only if he or she had not used any other lice treatment or home remedy within the past four weeks. Four subjects were each re-enrolled once in the study. Children were excluded if they had no hair, extremely short hair, a chronic scalp disorder, or were currently taking an antibiotic. Nurses were instructed to ask the parent/guardian about antibiotic use.

Once children were enrolled, guardians were given a copy of the product use directions, the test product, and a lice removal comb. The guardian treated the child at home with the product according to the manufacturer's instructions. Treatment procedures were as follows: First, apply the product to dry hair, then wait $10 \mathrm{~min}$. Next, with product still in the hair, separate hair into small sections and comb hair to remove lice and eggs. LiceMD ${ }^{\circ}$ products contain a patented comb specially designed to remove lice and their eggs from hair [30]. Finally, shampoo hair thoroughly with regular shampoo and warm water. After treating the child, the guardian completed the "First Application" Parent Diary and Observation Form in the treatment log. 
The first school day following the initial application of dimethicone, the school nurse examined the subject, checking for live lice and viable eggs while visually inspecting the condition of the child's scalp. If any live lice were found (or if the child had nits and the child's school had a "no nits" policy), the guardian was notified and advised to immediately apply a second dimethicone treatment. If a second application was done, the guardian was asked to complete the "Second Application" Parent Diary and Observation Form. If any scalp condition score was higher (worse) than the baseline score, the child was checked daily on school days until the irritation resolved. If a third application was needed, the same procedure was followed, and the guardian was asked to complete the "Third Application" page of the treatment log.

The guardian was asked to re-inspect the child's scalp and hair each day for lice, eggs and nits for 13 days. If lice or viable eggs were seen, the parent was asked to notify the school nurse. The school nurse confirmed the presence and instructed the parent to treat.

On all subjects, seven days ( \pm 2 days) and fourteen days ( \pm 2 days) after the initial application of treatment, the school nurse conducted a head check for lice and eggs and completed an Egg/Lice/Scalp Condition Form. If the nurse noticed any live lice, the guardian was notified and asked to do another treatment.

Fourteen days ( \pm 2 days) after the first treatment, the school nurse conducted another head check on the treated child and completed another Egg/Lice/Scalp Condition Form. If there was still a live lice infestation, the school nurse notified the guardian and discussed further treatment options. Guardians then completed the fourteen day product use questionnaire.

To comply with best practices in ethical approval and consent, this research study was conducted in accordance with the Declaration of Helsinki and was approved prior to the commencement by the HackensackUMC Institutional
Review Board (HIRB protocol number Pro00000685). Informed consent was obtained from each participant's parent or guardian prior to enrollment. The signed consent document included a statement informing parents/guardians that their child's identity will remain confidential if the results of the trial are published.

The protocol and clinical dataset will be made available upon request to any scientist wishing to use them for non-commercial purposes.

For the statistical analysis, the live lice and viable egg data were analyzed separately, using logistic regression to model the presence or absence of lice/eggs as a function of time, defined by days of treatment, and the other subject characteristics. The correlation between lice/egg counts for each subject on different days was accounted for by using a Generalized Estimating Equation (GEE) approach.

\section{Results}

\section{Demographics}

A total of 58 subjects were included in the study. Subject characteristics included age, gender, hair amount (thickness), hair length and number of applications. The subjects were aged between 3.5 and 13 years old, with a mean of nearly 7 years (6.98) and a standard deviation of 1.73, as summarized in Fig. 1. Although study eligibility called for subjects ages 3-12, one 13 year old student was inadvertently enrolled. Statistical analysis showed that age did not affect the rate of decrease in the presence of viable eggs due to treatment, i.e. there was no difference in the effectiveness of the treatment with age.

Definitions for hair amount and hair length were not supplied on the questionnaire and were determined by the school nurse and/or the parent filling out the form with the nurse's assistance. The inclusion of these characteristics was mainly to explore the possibility of varying product effectiveness with these characteristics.

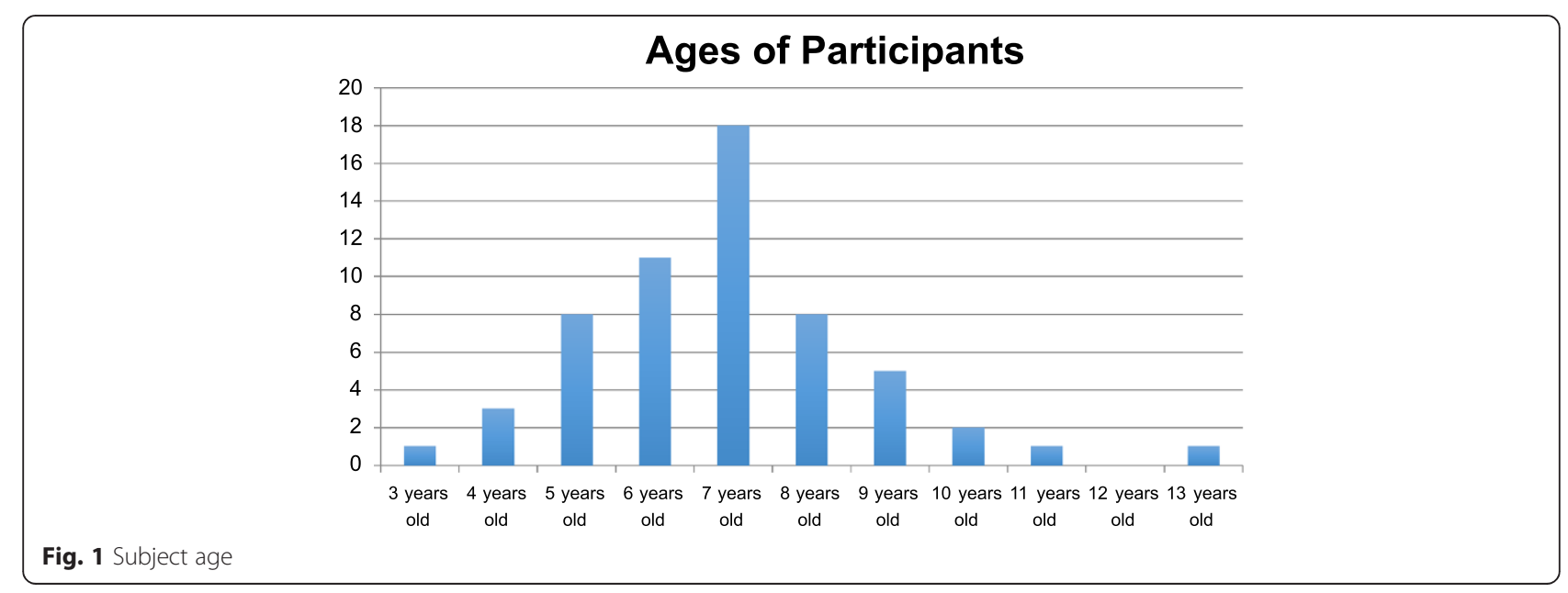


No significant differences in effectiveness were found with respect to gender, age or hair characteristics. Table 1 displays summary statistics of the data.

We enrolled 97 subjects from six schools, resulting in 58 evaluable charts. The 39 subjects not evaluable were excluded due to reasons detailed in Table 2, including issues with documentation (incomplete or missing study forms) or not meeting inclusion criteria.

The study included a comparison group in which those opting to use a treatment other than LiceMD could enroll and still be followed for outcomes according to the same criteria in the experimental arm. All subjects chose to enroll in the experimental arm of the study.

\section{Efficacy}

The data consist of counts of live lice and viable eggs found in 58 subjects at baseline (Day 0) and on Day 1, Day 7, and Day 14 after treatment as shown in Fig. 2. After one day of treatment, $98.30 \%$ of subjects (57 of 58 ) were free of live lice and $55.20 \%$ (32 of 58) were free of viable eggs. At diagnosis, 55 subjects had viable eggs with three subjects meeting enrollment criteria for three or more live lice. On day 14 of the study, $96.50 \%$ of subjects (55 of 57) were still free of live lice, and $80.70 \%$ (46 of 57) were free of viable eggs. If a child was still found with live lice on day 14, the school nurse notified the guardian to discuss further treatment options, as subjects could not immediately re-enroll in the study. The removal of eggs was facilitated by the viscosity of the product and the lice comb provided, which together eased removal.

Of the 58 subjects, 43 received a total of one treatment, ten received two treatments and five received three treatments [Table 1]. Subjects received a second or third treatment if lice or viable eggs were confirmed by the school nurse.

Table 1 Subject characteristics

\begin{tabular}{llc}
\hline Variable & Values & Number of subjects \\
\hline Gender & Female & 45 \\
& Male & 13 \\
Hair amount & Thin & 14 \\
& Medium & 23 \\
& Thick & 20 \\
Hair length & Short & 15 \\
& Long & 38 \\
Final Number of treatment applications & 1 & 5 \\
& 2 & 43 \\
& 3 & 10 \\
\hline
\end{tabular}

Table 2 Subject exclusions

\begin{tabular}{lc}
\hline Reason & Number of participants \\
\hline Protocol was not Followed at Enrollment & 5 \\
Protocol was not Followed during Study & 1 \\
Documentation Issue at Enrollment & 1 \\
Documentation Issue during Study & 10 \\
Did not have Live Lice & 8 \\
Had another Treatment during the Study & 3 \\
Did not meet Inclusion Criteria & 6 \\
Did not Return to School & 5 \\
\hline
\end{tabular}

\section{Safety}

All subjects were monitored by the school nurse at baseline and throughout the study period for adverse effects, including scalp erythema, excoriation, flaking and edema. One adverse event was reported by a parent/guardian during the study, which was transcribed by the school nurse on the Adverse Event Report form as "Irritation on cheek at time of shampoo application" which occurred "10 min after shampoo was washed off." The nurse also reported that the irritation lasted $10 \mathrm{~min}$ and that no medical attention was needed. There were no reports of significant adverse events during the study. Overall, scalp conditions as assessed by the school nurse improved during the two week study period: 10 subjects $(17.5 \%)$ reported mild to moderate scalp erythema on day 1 , compared with only one subject $(1.7 \%)$ on day $14 ; 8$ subjects $(14.3 \%)$ reported mild scalp excoriation on day 1 , with none reporting on day 14 .

\section{Discussion}

This study demonstrates the efficacy and short-term safety for the topical use of $100 \%$ dimethicone for treating pediatric pediculosis. While prior studies have shown efficacy for dimethicone-containing products, to our knowledge this is the first study to document the in vivo safety and efficacy for $100 \%$ dimethicone. Additionally, this study design utilized school and daycare settings to evaluate children for head lice, which is of practical importance as many children with lice are identified and followed by school nurses. There were several limitations for this study: this was an open label single arm design with no comparison group. Additionally, this study had a short term assessment for adverse events.

\section{Limitations}

Originally enrollment was estimated to be 200 subjects, as determined by a questionnaire distributed to the school nurses, asking the approximate number of head lice cases per school year. The study team then estimated the number of expected cases presenting at each participating school during the anticipated enrollment 


\section{Subjects with/without lice eggs}

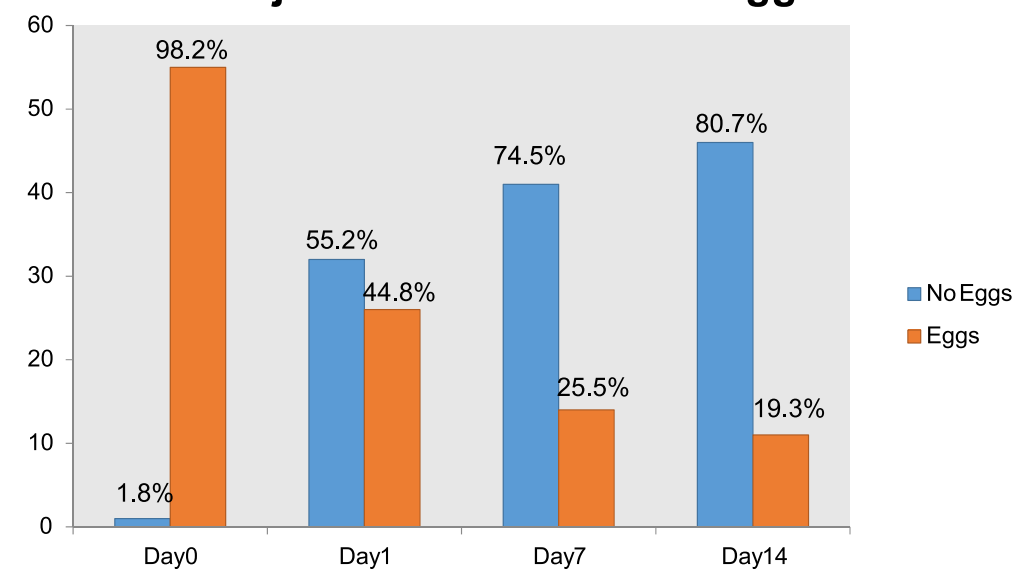

Subjects with/without live lice

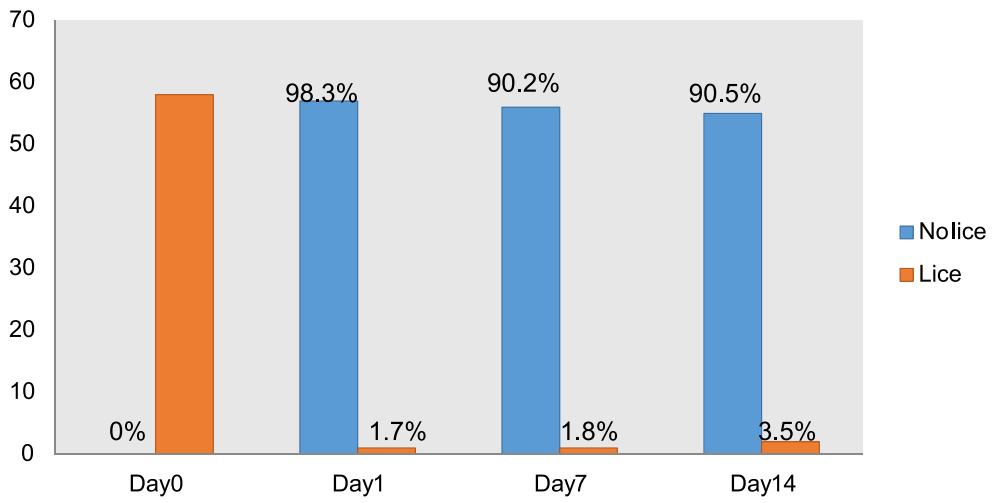

Fig. 2 Efficacy of $100 \%$ dimethicone treatment

period from May 2009 through June 2011. This enrollment period was later extended to enroll additional subjects, because only six schools out of 19 actively enrolled subjects and the number of subjects enrolled at each school was less than anticipated. School nurses encountered multiple challenges including limitations with communicating instructions and follow-up with the parents/guardians. The nurses also encountered time restrictions due to high student volume in some schools, which at times proved challenging to complete the necessary amount of study paperwork. The study necessitated many volunteer hours from participating school nurses. Efforts to keep school nurses engaged in the study and reminded of protocol procedures included regular e-newsletters and updates, phone calls and in-person visits by the study coordinator and research nurse to assess needs and answer questions.

Study enrollment concluded in June 2013. Funding restrictions limiting enrollment time occurred in December 2010 when the sponsor was purchased by another company and discontinued funding this research. At that time, The Deirdre Imus Environmental Health Center ${ }^{\odot}$ at Hackensack University Medical Center continued needed funding until the study's conclusion.

\section{Conclusions}

A $100 \%$ dimethicone product is a safe and highly effective head lice treatment for children and may serve as a potentially less toxic and less resistance prone alternative to pesticide-containing products. Given its safety and efficacy record, dimethicone should be considered as a first-line treatment for pediatric head lice.

\section{Competing interests}

The authors have no competing financial interests to declare. This work was funded in part by Combe Incorporated, White Plains, NY, which contributed the study drug and helped draft the protocol. Combe, Inc. was not involved in the collection, analysis or interpretation of data, nor in the writing of the manuscript or decision to submit it for publication. The study was also funded by The Deirdre Imus Environmental Health Center $^{\oplus}$ (DIEHC) at Hackensack University Medical Center, which carried out this study. Sources of funding for each author: El, LR and JB are employees of HackensackUMC; JML was paid as a statistical consultant by DIEHC. 


\section{Authors' contributions}

ESI participated in study oversight, nurse training and drafting and revision of the manuscript. JB participated in conceptualizing the study, contributed medical expertise and detailed manuscript review. JML performed oversight of the statistical analysis and data interpretation. LR performed study design oversight, contributed medical expertise, participated in manuscript preparation and detailed manuscript review. All authors read and approved the final manuscript.

\section{Acknowledgements}

The research team would like to thank the school nurses, Research Nurse Karen Overgaard, as well as interns Nikita Manavi, Lucerito Ruiz and Micaela Itona for their assistance and dedication.

\section{Dataset}

Permission to use the dataset was granted by LR and ESI. The dataset is available upon request to any scientist wishing to use it for non-commercial purposes.

\section{Author details}

${ }^{1}$ The Deirdre Imus Environmental Health Center ${ }^{\oplus}$, Hackensack University Medical Center, 30 Prospect Ave, Hackensack, NJ 07601, USA. ${ }^{2}$ Hackensack University Medical Center, The Joseph M. Sanzari Children's Hospital, 30 Prospect Avenue, Hackensack, NJ 07601, USA. ${ }^{3}$ Dept. of Mathematical Sciences, NJ Institute of Technology - University Heights, Newark, NJ 07102, USA.

Received: 25 August 2014 Accepted: 18 May 2015

Published online: 20 June 2015

\section{References}

1. Centers for Disease Control \& Prevention Head Lice FAQs - Who is at Risk for Getting Head Lice? [http://www.cdc.gov/parasites/lice/head/gen_info/ faqs.html]

2. Frankowski BL. Clinical Report: Head Lice. Pediatrics. 2010;126(2):392-403.

3. Pickering LK. Pediculosis Capitis (Head Lice). In: Red Book: 2012 Report of the Committee on Infectious Diseases. Elk Grove Village, IL: American Academy of Pediatrics; 2012. p. 543-6.

4. Carozza SE, Li B, Elgethun K, Whitworth R. Risk of Childhood Cancers Associated with Residence in Agriculturally Intense Areas in the United States. Environ Health Perspect. 2008;116(4):559-65.

5. Ferreira JD, Couto AC, Pombo-de-Oliveira MS, Koifman S. In Utero Pesticide Exposure and Leukemia in Brazilian Children $<2$ Years of Age. Environ Health Perspect. 2013:121(2):269-75.

6. Naeher LP, Barr DB, Rithmire N, Edwards J, Holmes AK, Needham LL, et al. Pesticide Exposure Resulting from Treatment of Lice Infestation in School-Aged Children in Georgia. Environ Int. 2009;35(2):358-62.

7. Durand R, Bouvresse S, Berdjane Z, Izri A, Chosidow O, Clark JM. Insecticide resistance in head lice: clinical, parasitological and genetic aspects. Clin Microbiol Infect. 2012;18(4):338-44.

8. Clark JM. Determination, mechanism and monitoring of knockdown resistance in permethrin-resistant human head lice, Pediculus humanus capitis. J Asia Pac Entomol. 2009;12(1):1-7.

9. Bouvresse S, Berdjane Z, Durand R, Bouscaillou J, Izri A, Chosidow O. Permethrin and malathion resistance in head lice: results of ex vivo and molecular assays. J Am Acad Dermatol. 2012;67(6):1143-50.

10. Chosidow O, Chastang C, Brue C, Bouvet E, Izri M, Monteny N. Controlled study of malathion and d-phenothrin lotions for Pediculus humanus var capitis-infested schoolchildren. Lancet. 1994;344(8939-8940):1724-7.

11. Rupes V, Moravec J, Chmela J, Ledvinka J, Zelenková J. A resistance of head lice (Pediculus capitis) to permethrin in Czech Republic. Cent Eur J Public Health. 1995;3(1):30-2.

12. Mumcuoglu KY, Hemingway J, Miller J, loffe-Uspensky I, Klaus S, Ben-Ishai F, et al. Permethrin resistance in the head louse Pediculus capitis from Israel. Med Vet Entomol. 1995;9(4):427-32. 447

13. Burgess IF. The mode of action of dimeticone $4 \%$ lotion against head lice. Pediculus capitis BMC Pharmacol. 2009;9:3.

14. Burgess IF, Brunton ER, Burgess NA. Single application of $4 \%$ dimeticone liquid gel versus two applications of $1 \%$ permethrin creme rinse for treatment of head louse infestation: a randomised controlled trial. BMC Dermatol. 2013;13:5.
15. Heukelbach J, Pilger D, Oliveira FA, Khakban A, Ariza L, Feldmeier H. A highly efficacious pediculicide based on dimeticone: randomized observer blinded comparative trial. BMC Infect Dis. 2008;8:115.

16. Burgess IF, Lee PN, Matlock G: Randomised, controlled, assessor blind trial comparing $4 \%$ dimeticone lotion with $0.5 \%$ malathion liquid for head louse infestation. PLoS One 2007,2(11):e1127.

17. Burgess IF. Head Lice. Clin Evid. 2011;05:1703.

18. Heukelbach J, Sonnberg S, Becher H, Melo I, Speare R, Oliveira FA. Ovicidal efficacy of high concentration dimeticone: a new era of head lice treatment. J Am Acad Dermatol. 2011;64(4):e61-2.

19. Burgess IF, Burgess NA. Dimeticone $4 \%$ liquid gel found to kill all lice and eggs with a single 15 minute application. BMC Res Notes. 2011;4:15.

20. Izri A, Uzzan B, Maigret M, Gordon MS, Bouges-Michel C. Clinical efficacy and safety in head lice infection by Pediculus humanis capitis De Geer (Anoplura: Pediculidae) of a capillary spray containing a silicon-oil complex. Parasite. 2010;17(4):329-35.

21. Kurt O, Balcioğlu IC, Burgess IF, Limoncu ME, Girginkardeșler N, Tabak T, et al. Treatment of head lice with dimeticone $4 \%$ lotion: comparison of two formulations in a randomised controlled trial in rural Turkey. BMC Public Health. 2009;9:441.

22. Heukelbach J, Pilger D, Oliveira FA, Khakban A, Ariza L, Feldmeier H. A highly efficacious pediculicide based on dimeticone: randomized observer blinded comparative trial. BMC Infect Dis. 2008;8:115.

23. Burgess IF, Brown CM, Lee PN. Treatment of head louse infestation with $4 \%$ dimeticone lotion: randomised controlled equivalence trial. BMJ. 2005:330(7505):1423.

24. Heukelbach J, Asenov A, Liesenfeld O, Mirmohammadsadegh A, Oliveira FA. A new two-phase dimeticone pediculicide shows high efficacy in a comparative bioassay. BMC Dermatol. 2009;9:12.

25. Gallardo A, Mougabure-Cueto G, Vassena C, Picollo MI, Toloza AC. Comparative efficacy of new commercial pediculicides against adults and eggs of Pediculus humanus capitis (head lice). Parasitol Res. 2012;110(5):1601-6.

26. Strycharz JP, Lao AR, Alves AM, Clark JM. Ovicidal response of NYDA formulations on the human head louse (Anoplura: Pediculidae) using a hair tuft bioassay. J Med Entomol. 2012:49(2):336-42.

27. Speare R. A single application of dimeticone is superior to two applications of permethrin in ridding head lice. J Pediatr. 2013:163(5):1531-2.

28. Committee on Infectious Diseases, American Academy of Pediatrics. Pickering LK, ed. American Academy of Pediatrics Red Book ${ }^{\oplus}: 2012$ Report of the Committee on Infectious Diseases - Pediculosis Capitis. 2012; 29th Ed:543-46.

29. Devore, CD, Schutze, GE and the Council on School Health and Committee on Infectious Diseases. American Academy of Pediatrics Clinical Report: Head Lice. Pediatrics. 2015; 135:(5) e1355-e1365; published ahead of print April 27, 2015

30. Getting Rid of Lice - LiceMD ${ }^{\circledast}$ Pesticide Free Treatment Instructions [http://www.licemd.com/getting-rid-of-lice.php]

\section{Submit your next manuscript to BioMed Central and take full advantage of:}

- Convenient online submission

- Thorough peer review

- No space constraints or color figure charges

- Immediate publication on acceptance

- Inclusion in PubMed, CAS, Scopus and Google Scholar

- Research which is freely available for redistribution 